\title{
Boundary characteristic orthogonal polynomials in the study of transverse vibrations of nonhomogeneous rectangular plates with bilinear thickness variation
}

\author{
R. Lal* and Yajuvindra Kumar \\ Department of Mathematics, Indian Institute of Technology Roorkee, Roorkee, India
}

Received 6 June 2009

Revised 15 January 2011

\begin{abstract}
The free transverse vibrations of thin nonhomogeneous rectangular plates of variable thickness have been studied using boundary characteristic orthogonal polynomials in the Rayleigh-Ritz method. Gram-Schmidt process has been used to generate these orthogonal polynomials in two variables. The thickness variation is bidirectional and is the cartesian product of linear variations along two concurrent edges of the plate. The nonhomogeneity of the plate is assumed to arise due to linear variations in Young's modulus and density of the plate material with the in-plane coordinates. Numerical results have been computed for four different combinations of clamped, simply supported and free edges. Effect of the nonhomogeneity and thickness variation with varying values of aspect ratio on the natural frequencies of vibration is illustrated for the first three modes of vibration. Three dimensional mode shapes for all the four boundary conditions have been presented. A comparison of results with those available in the literature has been made.
\end{abstract}

Keywords: Nonhomogeneous, rectangular, bilinear thickness, Rayleigh-Ritz

\section{Introduction}

Plates of various geometries are widely used as structural components in various technological situations ranging from household goods such as mixer, grinder etc. to modern space technology. By appropriate variation of plate thickness, these plates can have significantly greater efficiency for vibration as compared to the plates of uniform thickness and also provide the advantage of reduction in weight and size. Numerous studies have been made by researchers on free vibrations of rectangular plates with different types of thickness variations and boundary conditions. The work up to 1985 has been compiled by Leissa in his monograph [1] and a series of review articles [25]. The thickness variations are mainly unidirectional such as linear, parabolic, stepped, quadratic and exponential etc. The analysis has been presented using analytical, numerical or approximate methods. Notable contributions, made thereafter are listed in references [6-15]. In this context, authors have come across a limited number of papers in which the thickness of the plate varies in both the directions and reported in references [16-23], to mention a few. Out of these, Sakiyama and Huang [19] considered sinusoidal variation, Cheung and Zhou [20] assumed the power functions of both the coordinates and the rest deal with bilinear variation in thickness.

\footnotetext{
${ }^{*}$ Corresponding author. Tel.: +91 1332 285151; Fax: +91 1332 273560; E-mail: rlatmfma@iitr.ernet.in.
} 
Nonhomogeneous elastic plates find their applications in the design of space vehicle, modern missile and aircraft wings. Various models for the nonhomogeneity of the plate material are available in the literature and a brief review is given in reference [24]. Recently, a number of papers have appeared in the literature analyzing the effect of nonhomogeneity on the vibrational behavior of rectangular plates and listed in references [25-27]. In these studies, it has been assumed that nonhomogeneity of the plate material i.e. material properties are functions of only one variable. However, in many practical situations, particularly in modern missile technology and microelectronics, plate type structural components have to work under high temperature conditions, which cause nonhomogeneity. For such type of situations, it is more appropriate to take the variation in the mechanical properties of the material as the function of two variables instead of one. During the survey of literature, it has been found that (i) no work has been done dealing with the vibration of rectangular plates in which the nonhomogeneity of the plate material depends upon the in-plane variables (ii) the boundary characteristic orthogonal polynomials have been extensively used for analyzing the dynamic behavior of the plates of various geometries under different boundary conditions. The simplification of the eigenvlaue problem and the rapid convergence are the main characteristics of the approach.

Keeping in view the above fact, a study dealing with nonhomogeneous rectangular plates of varying thickness using two dimensional boundary characteristic orthogonal polynomials in the Rayleigh-Ritz method is presented employing classical plate theory. These orthogonal polynomials may be generated either by Gram-Schmidt process [28] or using the recurrence relation [29]. In the present work, these orthogonal polynomials are generated using the Gram-Schmidt process which has an advantage over the procedure proposed by Liew et al. [30] and Bhat [31] that all the polynomials will automatically satisfy the essential boundary conditions. Further, this procedure to generate orthogonal polynomials for a particular boundary condition need not be repeated for various values of aspect ratio of the plate. Thus the present methodology saves time and efforts. Nonhomogeneity is assumed to arise due to linear variations in Young's modulus and density of the plate material with both the in-plane variables. The value of Poisson ratio is assumed to remain constant. The thickness of the plate is varying bidirectionally and is the cartesian product of linear variations along the two concurrent edges of the plate. The above considerations give-rise seven plate parameters and study of their effects on the natural frequencies for all the possible 21 combinations of classical boundary conditions at the four edges generates the huge data. In the present work, only four different combinations of edge conditions namely: CCCC- all the four edges are clamped; SCSC- two opposite edges are clamped and other two are simply supported; FCFC- two opposite edges are clamped and other two are free; FSFS- two opposite edges are simply supported and the other two are free have been considered. Mode shapes for the first three frequencies of a specified plate have been plotted. A comparison of results has been presented.

\section{Formulation and solution of the problem}

Consider an isotropic nonhomogeneous rectangular plate of varying thickness $h(x, y)$ occupying the domain 0 $\leqslant x \leqslant a, 0 \leqslant y \leqslant b$ in $x y$ - plane, where $a$ and $b$ are the length and the breadth of the plate, respectively. The $x-$ and $y$ - axes are taken along the edges of the plate and axis of $z$-is perpendicular to the $x y$ - plane. The middle surface being $z=0$ and origin is at one of the corners of the plate as shown in Fig. 1(a).

The expressions for strain energy and kinetic energy of the plate are given by

$$
\begin{aligned}
& V=\frac{1}{2} \int_{0}^{a} \int_{0}^{b} D\left[w_{x x}^{2}+2 v w_{x x} w_{y y}+w_{y y}^{2}+2(1-v) w_{x y}^{2}\right] d x d y \\
& T=\frac{1}{2} \int_{0}^{a} \int_{0}^{b} \rho h\left(\frac{\partial w}{\partial t}\right)^{2} d x d y,
\end{aligned}
$$

where $D=\frac{E h^{3}}{12\left(1-v^{2}\right)}$ is the flexural rigidity, $E(x, y)$ is the Young's modulus, $\rho(x, y)$ is the density, $v$ is the Poisson's ratio, $t$ is the time, $w(x, y, t)$ is the plate deflection at point $(x, y)$ and subscript denotes the partial differentiation with respect to that variables. 


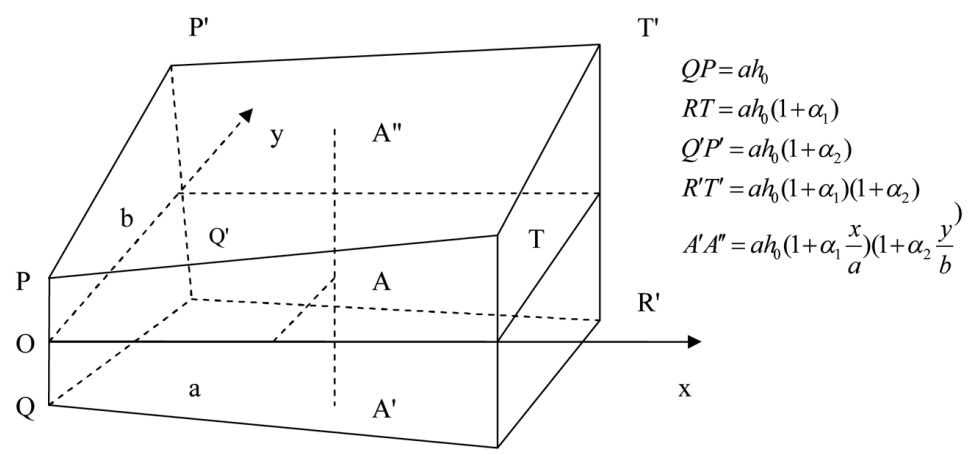

$\mathrm{R}$

(a)
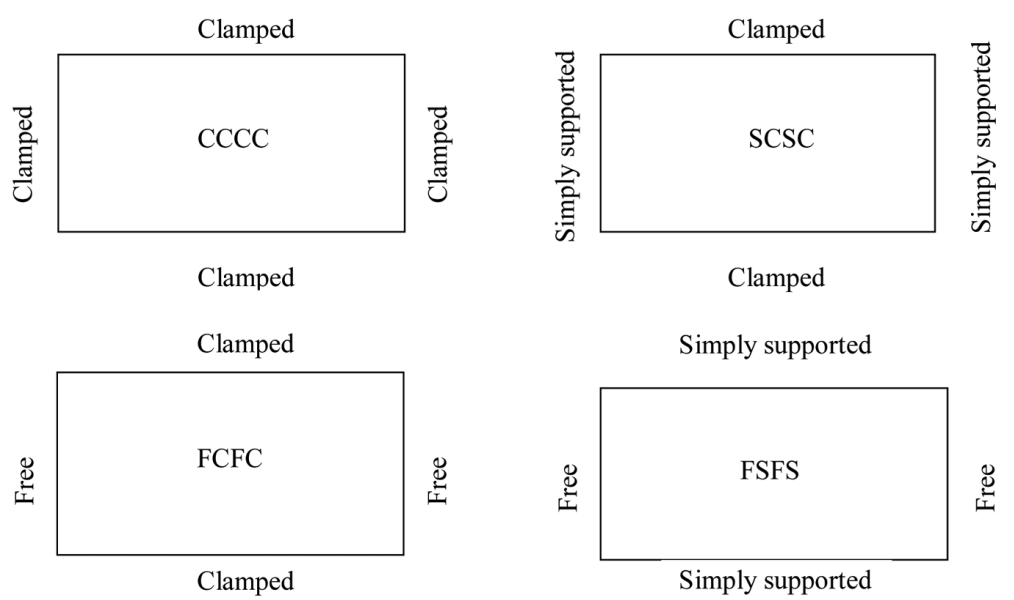

(b)

Fig. 1. (a) Geometry of the plate and (b) boundary conditions.

For harmonic solution, the deflection function is assumed to be

$$
w(x, y, t)=\bar{W}(x, y) \sin \omega t,
$$

where $\bar{W}(x, y)$ represents the maximum transverse displacement at the point $(x, y)$ and $\omega$ is the circular frequency.

Substituting relation (3) in expressions (1) and (2), the expressions for maximum strain energy and kinetic energy of the plate become

$$
\begin{aligned}
V_{\max } & =\frac{1}{2} \int_{0}^{a} \int_{0}^{b} D\left[\bar{W}_{x x}^{2}+2 v \bar{W}_{x x} \bar{W}_{y y}+\bar{W}_{y y}^{2}+2(1-v) \bar{W}_{x y}^{2}\right] d x d y \\
T_{\max } & =\frac{\omega^{2}}{2} \int_{0}^{a} \int_{0}^{b} \rho h \bar{W}^{2} d x d y .
\end{aligned}
$$

According to the Raleigh-Ritz method equating Eqs (4) and (5), one gets the Rayleigh quotient as

$$
\omega^{2}=\frac{\int_{0}^{a} \int_{0}^{b} D\left[\bar{W}_{x x}^{2}+2 v \bar{W}_{x x} \bar{W}_{y y}+\bar{W}_{y y}^{2}+2(1-v) \bar{W}_{x y}^{2}\right] d x d y}{\int_{0}^{a} \int_{0}^{b} \rho h \bar{W}^{2} d x d y} .
$$


Introducing the non-dimensional variables $X=x / a, Y=y / b, W=\bar{W} / a$ and $H=h / a$ together with the assumption that the Young's modulus and density of the plate material vary with the in-plane coordinates by the functional relations

$$
E=E_{0}\left(1+\alpha_{1} X+\alpha_{2} Y\right) \text { and } \rho=\rho_{0}\left(1+\beta_{1} X+\beta_{2} Y\right)
$$

and thickness of plate varies linearly in both $X$ - and $Y$ - directions given by

$$
H(H, Y)=h_{0}\left(1+\gamma_{1} X\right)\left(1+\gamma_{2} Y\right),
$$

where $E_{0}, \rho_{0}$ and $h_{0}$ are Young's modulus, density and thickness of the plate at $X=0, Y=0$, respectively.

Eq. (6) reduces to

$$
\omega^{2}=\frac{\int_{0}^{1} \int_{0}^{1} D_{0}\left[W_{X X}^{2}+2 v\left(\frac{a}{b}\right)^{2} W_{X X} W_{Y Y}+\left(\frac{a}{b}\right)^{4} W_{Y Y}^{2}+2(1-v)\left(\frac{a}{b}\right)^{2} W_{X Y}^{2}\right] d X d Y}{\int_{0}^{1} \int_{0}^{1} \rho_{0} h_{0}\left(1+\beta_{1} X+\beta_{2} Y\right)\left(1+\gamma_{1} X\right)\left(1+\gamma_{2} Y\right) W^{2} d X d Y},
$$

where $D_{0}=\frac{E_{0} h_{0}^{3}\left(1+\alpha_{1} X+\alpha_{2} Y\right)\left(1+\gamma_{1} X\right)^{3}\left(1+\gamma_{2} Y\right)^{3}}{12 a\left(1-v^{2}\right)}$.

Now satisfying the essential boundary conditions, let us assume

$$
W(X, Y)=\sum_{k=1}^{N} d_{k} \hat{\phi}_{k}(X, Y),
$$

where $N$ is the order of approximation to get the desired accuracy, $d_{k}$ 's are unknowns and $\hat{\phi}_{k}$ are orthonormal polynomials which are generated using Gram-Schmidt process as follows:

Orthogonal polynomials $\phi_{k}$ over the region $0 \leqslant X \leqslant 1,0 \leqslant Y \leqslant 1$ have been generated with the help of linearly independent set of functions $L_{k}=1 l_{k}, k=1,2,3, \ldots$, with

$$
l=X^{p_{1}}(1-X)^{p_{2}} Y^{p_{3}}(1-Y)^{p_{4}}, \quad l_{k}=\left\{1, X, Y, X^{2}, X Y, Y^{2}, X^{3}, X^{2} Y, X Y^{2}, Y^{3}, \ldots\right\},
$$

where $p_{1}=0,1$ or 2 as the edge $X=0$ is free, simply supported or clamped. Same justification can be given to $p_{2}$, $p_{3}$ and $p_{4}$ for the edges $X=1, Y=0$ and $Y=1$.

$$
\begin{aligned}
& \phi_{1}=L_{1}, \quad \phi_{k}=L_{k}-\sum_{j=1}^{k-1} \alpha_{k j} \phi_{j}, \\
& \alpha_{k j}=\frac{<L_{k}, \phi_{j}>}{<\phi_{j}, \phi_{j}>}, \quad j=1,2,3, \ldots,(k-1), \quad k=2,3,4, \ldots, N .
\end{aligned}
$$

The inner product of the functions say, $\phi_{1}$ and $\phi_{2}$ is defined as

$$
<\phi_{1}, \phi_{2}>=\int_{0}^{1} \int_{0}^{1}\left(1+\beta_{1} X+\beta_{2} Y\right)\left(1+\gamma_{1} X\right)\left(1+\gamma_{2} Y\right) \phi_{1}(X, Y) \phi_{2}(X, Y) d X d Y,
$$

where $\left(1+\beta_{1} X+\beta_{2} Y\right)\left(1+\gamma_{1} X\right)\left(1+\gamma_{2} Y\right)$ is the weight function and the norm of the function $\phi_{1}$ is given by

$$
\left\|\phi_{1}\right\|=<\phi_{1}, \phi_{1}>1 / 2=\left[\int_{0}^{1} \int_{0}^{1}\left(1+\beta_{1} X+\beta_{2} Y\right)\left(1+\gamma_{1} X\right)\left(1+\gamma_{2} Y\right) \phi_{1}^{2}(X, Y) d X d Y\right]^{1 / 2} .
$$

The normalization can be done by using

$$
\hat{\phi}_{k}=\frac{\phi_{k}}{\left\|\phi_{k}\right\|} \text {. }
$$


Using expression (10) into Eq. (9) and minimization of the resulting expression for $\omega^{2}$ w.r.t. $d_{k}$ 's leads to the standard eigenvalue problem

$$
\sum_{k=1}^{N}\left(a_{j k}-\Omega^{2} \delta_{j k}\right) d_{k}=0, j=1,2,3, \ldots, N
$$

where

$$
\begin{aligned}
& a_{j k}=\int_{0}^{1} \int_{0}^{1} F\left[\hat{\phi}_{j}^{X X} \hat{\phi}_{k}^{X X}+v \mu^{2}\left(\hat{\phi}_{j}^{X X} \hat{\phi}_{k}^{Y Y}+\hat{\phi}_{k}^{X X} \hat{\phi}_{j}^{Y Y}\right)+2(1-v) \mu^{2} \hat{\phi}_{j}^{X Y} \hat{\phi}_{k}^{X Y}+\mu^{4} \hat{\phi}_{j}^{Y Y} \hat{\phi}_{k}^{Y Y}\right] d X d Y,(16) \\
& F=\left(1+\alpha_{1} X+\alpha_{2} Y\right)\left(1+\gamma_{1} X\right)^{3}\left(1+\gamma_{2} Y\right)^{3}, \quad \Omega^{2}=\frac{12 \rho_{0} a^{2}\left(1-v^{2}\right) \omega^{2}}{E_{0} h_{0}^{2}}, \quad \mu=\frac{a}{b}
\end{aligned}
$$

and $\delta_{j k}=\left\{\begin{array}{ll}1, & \text { if } j=k \\ 0, & \text { if } j \neq k\end{array}\right.$.

The integrals involved in Eq. (16) have been evaluated using the formula

$$
\int_{0}^{1} \int_{0}^{1} X^{p_{1}}(1-X)^{p_{2}} Y^{p_{3}}(1-Y)^{p_{4}} d X d Y=\frac{p_{1} ! p_{2} ! p_{3} ! p_{4} !}{\left(p_{1}+p_{2}+1\right) !\left(p_{3}+p_{4}+1\right) !} .
$$

\section{Boundary conditions}

The four boundary conditions namely CCCC, SCSC, FCFC and FSFS have been considered in which C stands for clamped edge, $\mathrm{S}$ for simply supported edge and $\mathrm{F}$ for free edge. The edge conditions are taken in anti-clockwise direction starting at the edge $x=0$ (Fig. 1(b)) and obtained by assigning various values to $p_{1}, p_{2}, p_{3}$ and $p_{4}$ as 0,1 , 2 for free, simply supported and clamped edge conditions, respectively.

\section{Results and discussion}

The numerical values of the frequency parameter $\Omega$ have been obtained by solving Eq. (15) employing Jacobi method. The lowest three eigenvalues have been reported as the first three natural frequencies corresponding to different boundary conditions considered here. The values of various plate parameters for these three modes of vibration are taken as follows.

Nonhomogeneity parameters: $\alpha_{1}, \alpha_{2}, \beta_{1}, \beta_{2}=-0.5,-0.4,-0.3,-0.2,-0.1,0.0,0.1,0.2,0.3,0.4,0.5$, thickness parameters: $\gamma_{1}, \gamma_{2}=-0.5,-0.4,-0.3,-0.2,-0.1,0.0,0.1,0.2,0.3,0.4,0.5$, aspect ratio: $a / b=0.25,0.50,0.75$, $1.00,1.25,1.50,1.75,2.00$ and $v=0.3$.

To choose the appropriate value of the order of approximation $N$, a computer program developed in $\mathrm{C}++$ for the evaluation of frequency parameter $\Omega$ was run for different values of $N$. The numerical values showed a consistent improvement with the increasing value of $N$ for different sets of the values of plate parameters. Table 1 shows the convergence of frequency parameter $\Omega$ with $N$ for a particular set of plate parameters for all the four boundary conditions where possibly maximum value of $N$ was required for first three modes. The value of $N$ may be taken as 45 but for the safer side of the accuracy of four decimal places for all possible combinations of the values of various plate parameters it has been taken $N=47$. All the computations were carried out in double precision arithmetic without observing any kind of numerical instability as pointed out by [32].

A comparison of frequency parameter $\Omega$ for homogeneous $\left(\alpha_{1}=\alpha_{2}=\beta_{1}=\beta_{2}=0\right)$ square $(a / b=1)$ plate of uniform $\left(\gamma_{1}=\gamma_{2}=0\right)$ thickness obtained by Rayleigh-Ritz method [7,34,37], exact and approximate results using Ritz method [33], Differential quadrature method [11,36], Optimized Kantorovich method [35] and non-uniform thickness obtained by finite element method [6], Rayleigh-Ritz method [7,9], differential quadrature method [11], optimized Kantorovich method [35] has been presented in Table 2. A close agreement of results is obtained. 
Table 1

Convergence of frequency parameter $\Omega$ with $N$ for $\alpha_{1}=\alpha_{2}=\beta_{1}=\beta_{2}=\gamma_{1}=\gamma_{2}=$ 0.5 and $a / b=1$

\begin{tabular}{|c|c|c|c|c|c|c|c|}
\hline \multicolumn{4}{|c|}{$\mathrm{CCCC}$} & \multicolumn{4}{|c|}{ SCSC } \\
\hline \multicolumn{4}{|c|}{ mode } & \multicolumn{4}{|c|}{ Mode } \\
\hline$N$ & I & II & III & $N$ & I & II & III \\
\hline 10 & 55.3971 & 112.4090 & 113.0890 & 10 & 44.7853 & 86.2823 & 106.5780 \\
\hline 20 & 55.3880 & 111.9560 & 112.8570 & 20 & 44.7636 & 84.3675 & 105.8680 \\
\hline 30 & 55.3837 & 111.9530 & 112.8320 & 30 & 44.7633 & 84.3289 & 105.8460 \\
\hline 40 & 55.3834 & 111.9470 & 112.8270 & 40 & 44.7633 & 84.3276 & 105.8450 \\
\hline 45 & 55.3822 & 111.9460 & 112.8270 & 45 & 44.7632 & 84.3274 & 105.8450 \\
\hline 46 & 55.3822 & 111.9460 & 112.8270 & 46 & 44.7632 & 84.3274 & 105.8450 \\
\hline 47 & 55.3822 & 111.9460 & 112.8270 & 47 & 44.7632 & 84.3274 & 105.8450 \\
\hline \multicolumn{4}{|c|}{ FCFC } & \multicolumn{4}{|c|}{ FSFS } \\
\hline \multicolumn{4}{|c|}{ mode } & \multicolumn{4}{|c|}{ mode } \\
\hline$N$ & I & II & III & $N$ & I & II & III \\
\hline 10 & 33.3218 & 42.9404 & 73.0086 & 10 & 15.0639 & 26.1364 & 62.6908 \\
\hline 20 & 33.2861 & 42.6022 & 68.4567 & 20 & 14.8862 & 25.6528 & 57.8629 \\
\hline 30 & 33.2634 & 42.5609 & 68.0796 & 30 & 14.8847 & 25.6334 & 57.4212 \\
\hline 40 & 33.2502 & 42.5341 & 68.0380 & 40 & 14.8845 & 25.6327 & 57.4015 \\
\hline 45 & 33.2455 & 42.5301 & 68.0372 & 45 & 14.8845 & 25.6325 & 57.3994 \\
\hline 46 & 33.2455 & 42.5301 & 68.0372 & 46 & 14.8845 & 25.6325 & 57.3994 \\
\hline 47 & 33.2455 & 42.5301 & 68.0372 & 47 & 14.8845 & 25.6325 & 57.3994 \\
\hline
\end{tabular}

To analyze the effect of various plate parameters on the frequencies, a huge amount of results are computed and all can not be given here. However, to observe their trends on the frequency parameter $\Omega$, some of them with their minimum and maximum values are reported in Tables 3-6 and few of them with their varying values are presented in Figs 2-5. It is observed that the frequency parameter $\Omega$ decreases in the order of boundary conditions CCCC $>$ SCSC $>$ FCFC $>$ FSFS for the same set of values of plate parameters. This may be attributed to the clamped edge condition which causes an additional constraint over simply supported and free edge conditions. Figure 2 shows the effect of nonhomogeneity parameter $\alpha_{1}$ on the frequency parameter $\Omega$ for $\beta_{1}= \pm 0.5, \gamma_{1}=0.5, \alpha_{2}= \pm 0.5$, $\beta_{2}=0.5, \gamma_{2}=0.5$ and $a / b=1$ for the first two modes of vibration. It is observed that the frequency parameter $\Omega$ increases with increasing values of $\alpha_{1}$ for all the boundary conditions keeping other plate parameters fixed. This may be attributed to the increased stiffness of the plate towards the edge $X=1$. The value of $\Omega$ decreases with the increasing values of $\beta_{1}$ while increases with the increasing value of $\alpha_{2}$ for fixed values of other plate parameters. The effect of $\beta_{1}$ and $\alpha_{2}$ is more pronounced for $\alpha_{1}=-0.5$ as compared to $\alpha_{1}=0.5$. The rate of increase in $\Omega$ with $\alpha_{1}$ is in the order of the boundary conditions CCCC $>$ SCSC $>$ FSFS $>$ FCFC when $\beta_{1}$ changes from -0.5 to 0.5 and order becomes CCCC $>$ SCSC $>$ FCFC $>$ FSFS when $\alpha_{2}$ changes from -0.5 to 0.5 , other parameters being fixed. This rate is higher in the second mode as compared to the first mode.

Figure 3 depicts the behavior of the frequency parameter $\Omega$ with the density parameter $\beta_{1}$ for $\alpha_{1}= \pm 0.5, \gamma_{1}=$ $0.5, \alpha_{2}=0.5, \beta_{2}= \pm 0.5, \gamma_{2}=0.5$ and $a / b=1$ for the first two modes of vibration. It is seen that the frequency parameter $\Omega$ decreases with increasing value of $\beta_{1}$ whatever be the values of other plate parameters. This may be due to the increased inertia of the plate towards the edge $X=1$. The frequency parameter $\Omega$ is found to increase with the increasing value of $\alpha_{1}$ keeping other plate parameters fixed. The value of $\Omega$ further decreases with the increasing value of $\beta_{2}$. The rate of increase in $\Omega$ with $\beta_{1}$ is in the order of the boundary conditions $C C C C>$ SCSC $>$ FSFS $>$ FCFC when $\alpha_{1}$ changes from -0.5 to 0.5 and order becomes CCCC $>$ SCSC $>$ FCFC $>$ FSFS when $\beta_{2}$ changes from -0.5 to 0.5 . This rate is more pronounced in the second mode as compared to the first mode.

Figure 4 demonstrates the effect of thickness parameter $\gamma_{1}$ on the frequency parameter $\Omega$ for $\alpha_{1}= \pm 0.5, \alpha_{2}=$ $0.5, \beta_{1}=0.5, \beta_{2}=0.5, \gamma_{2}= \pm 0.5$ and $a / b=1$ for the first two modes of vibration. It is noticed that the frequency parameter $\Omega$ increases with increasing value of $\gamma_{1}$ for all the boundary conditions for fixed values of all other plate parameters and this may happen due to further increase in the stiffness of the plate. The value of $\Omega$ increases with the increasing value of $\alpha_{1}$ as well as $\gamma_{2}$. The effect of $\gamma_{2}$ is more pronounced for $\gamma_{1}=0.5$ as compared to $\gamma_{1}=-0.5$ keeping all other parameters fixed. The rate of increase in $\Omega$ with $\gamma_{1}$ is in the order of the boundary conditions CCCC $>$ SCSC $>$ FSFS $>$ FCFC when $\alpha_{1}$ changes from -0.5 to 0.5 and order becomes $C C C C>$ SCSC $>$ FCFC $>$ FSFS when $\gamma_{2}$ changes from -0.5 to 0.5 , other parameters being fixed. This rate is higher in the second mode as compared to the first mode. 
Table 2

Frequency parameter $\Omega$ for CCCC plate

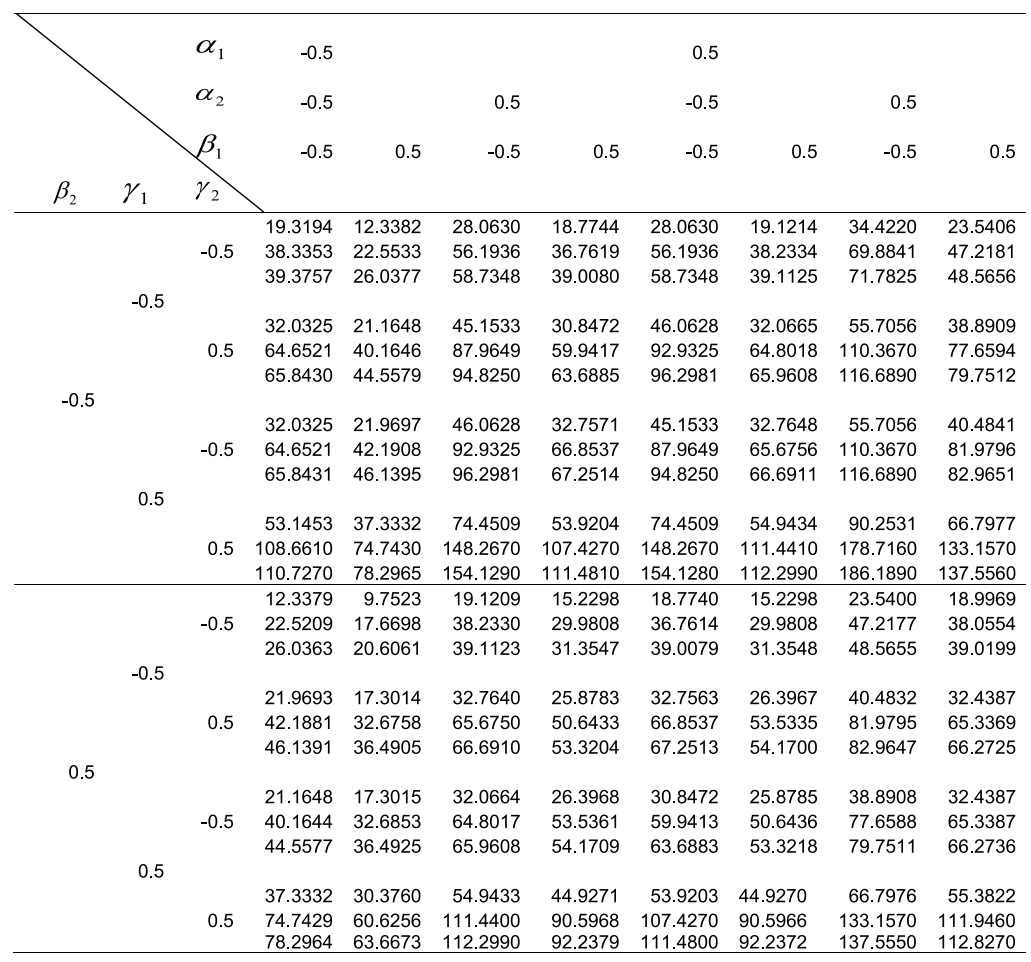

Table 3

Frequency parameter $\Omega$ for SCSC plate

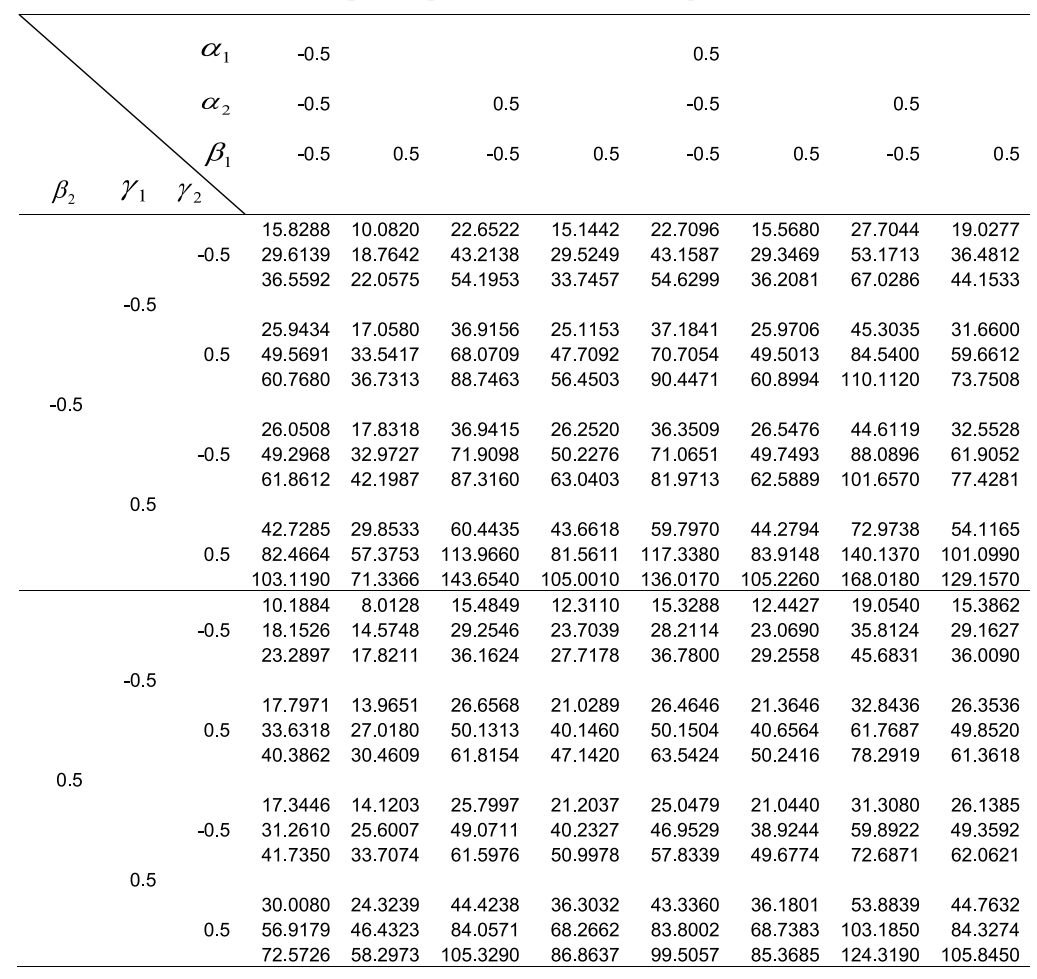


Table 4

Frequency parameter $\Omega$ for FCFC plate

\begin{tabular}{|c|c|c|c|c|c|c|c|c|c|c|}
\hline \multirow[b]{4}{*}{$\beta_{2}$} & \multirow[b]{4}{*}{$\gamma_{1}$} & $\alpha_{1}$ & -0.5 & \multicolumn{7}{|c|}{0.5} \\
\hline & & $\alpha_{2}$ & -0.5 & \multicolumn{3}{|c|}{0.5} & -0.5 & \multicolumn{3}{|c|}{0.5} \\
\hline & & & -0.5 & 0.5 & -0.5 & 0.5 & -0.5 & 0.5 & -0.5 & 0.5 \\
\hline & & & & & & & & & & \\
\hline \multirow{15}{*}{-0.5} & \multirow{8}{*}{-0.5} & \multirow{4}{*}{-0.5} & 11.5938 & 5.9495 & 17.1726 & 9.5506 & 17.1904 & 10.9074 & 21.1602 & 13.0431 \\
\hline & & & 15.8680 & 11.7064 & 21.6663 & 16.8238 & 21.6613 & 15.6651 & 26.0459 & 19.4423 \\
\hline & & & 24.8016 & 13.6796 & 35.1407 & 23.6275 & 35.5142 & 24.1670 & 43.2027 & 29.8098 \\
\hline & & & & & & & & & & \\
\hline & & \multirow{3}{*}{0.5} & 18.3658 & 9.7841 & 28.0636 & 16.0122 & 28.1795 & 18.0746 & 34.9588 & 21.8494 \\
\hline & & & 25.8790 & 19.7354 & 35.6276 & 28.0021 & 34.8492 & 25.9548 & 42.4493 & 32.4135 \\
\hline & & & 40.4169 & 23.2072 & 56.2973 & 39.3190 & 57.4089 & 40.1568 & 69.3879 & 49.1784 \\
\hline & & \multirow{4}{*}{-0.5} & & & & & & & & \\
\hline & \multirow{7}{*}{0.5} & & 19.1213 & 12.3241 & 25.7368 & 19.7460 & 23.0993 & 19.8592 & 28.6621 & 24.4745 \\
\hline & & & 24.9502 & 17.1471 & 37.8406 & 24.1452 & 41.1680 & 25.2953 & 49.6063 & 30.4764 \\
\hline & & & 40.5032 & 26.9577 & 58.9624 & 40.0693 & 57.9644 & 40.5369 & 73.5290 & 49.6994 \\
\hline & & & & & & & & & & \\
\hline & & \multirow{3}{*}{0.5} & 31.1564 & 20.2531 & 42.7561 & 33.1750 & 37.8281 & 32.8156 & 47.4944 & 40.8913 \\
\hline & & & 39.7283 & 28.1449 & 61.7380 & 40.3451 & 67.0782 & 41.9643 & 80.8927 & 50.9164 \\
\hline & & & 65.5782 & 45.2538 & 94.7201 & 65.9686 & 96.8160 & 67.4040 & 120.5530 & 81.9310 \\
\hline \multirow{15}{*}{0.5} & \multirow{8}{*}{-0.5} & \multirow{4}{*}{-0.5} & 6.6863 & 4.7972 & 10.9923 & 7.9145 & 11.7465 & 8.9028 & 14.4298 & 10.7474 \\
\hline & & & 10.8111 & 9.1740 & 15.3778 & 13.4101 & 14.0625 & 12.2173 & 17.5677 & 15.3714 \\
\hline & & & 15.0069 & 11.1011 & 23.8493 & 19.6690 & 23.0939 & 18.9916 & 28.9155 & 23.7650 \\
\hline & & & & & & & & & & \\
\hline & & \multirow{3}{*}{0.5} & 11.3965 & 8.0962 & 19.0827 & 13.6040 & 20.1149 & 15.1186 & 24.9737 & 18.4486 \\
\hline & & & 18.6459 & 15.9146 & 26.5452 & 23.0212 & 24.0450 & 20.8516 & 30.3209 & 26.4127 \\
\hline & & & 26.6934 & 19.2283 & 41.0383 & 33.3718 & 40.1307 & 32.7542 & 49.8699 & 40.6886 \\
\hline & & \multirow{4}{*}{-0.5} & & & & & & & & \\
\hline & \multirow{7}{*}{0.5} & & 13.0711 & 9.9391 & 18.8500 & 16.0805 & 16.7944 & 15.4351 & 21.1870 & 19.3335 \\
\hline & & & 15.3395 & 13.2940 & 24.5523 & 19.2691 & 26.6514 & 20.3939 & 32.5960 & 24.7882 \\
\hline & & & 25.4455 & 21.0139 & 39.4285 & 32.1096 & 39.5623 & 31.9591 & 49.3156 & 39.7811 \\
\hline & & & & & & & & & & \\
\hline & & \multirow{3}{*}{0.5} & 22.2239 & 16.7170 & 32.5837 & 27.6989 & 28.6241 & 26.2737 & 36.5184 & 33.2455 \\
\hline & & & 25.9767 & 22.4715 & 42.5456 & 33.1621 & 45.9160 & 34.7706 & 56.3468 & 42.5301 \\
\hline & & & 44.4380 & 36.4946 & 67.9009 & 54.8482 & 68.9300 & 55.1751 & 85.0350 & 68.0372 \\
\hline
\end{tabular}

Table 5

Frequency parameter $\Omega$ for FSFS plate

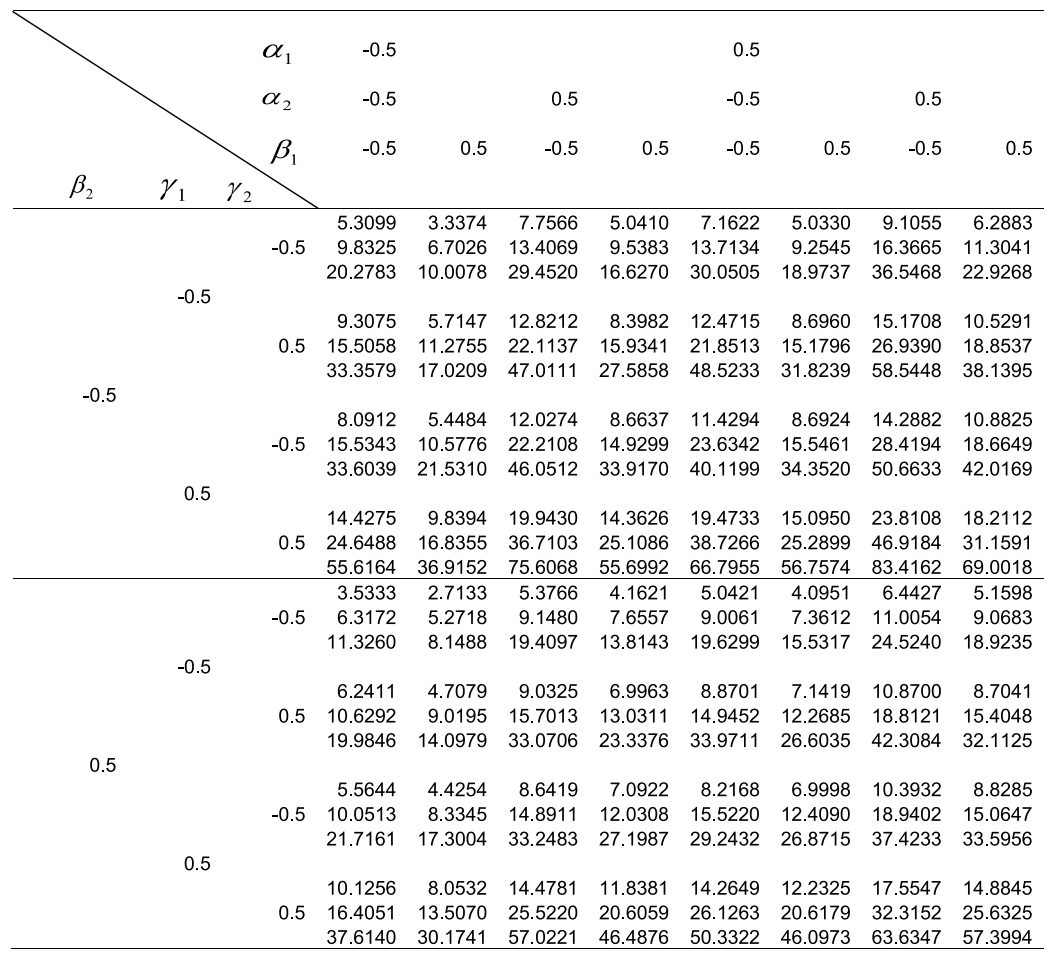


Table 6

Comparison of frequency parameter $\Omega$ for homogeneous $\left(\alpha_{1}=\alpha_{2}=\beta_{1}=\beta_{2}=\right.$ $0)$ square $(a / b=1)$ plate for $\gamma_{2}=0$ and $v=0.3$

\begin{tabular}{|c|c|c|c|c|c|}
\hline \multirow[t]{2}{*}{ Boundary Conditions } & \multirow[t]{2}{*}{ Ref. } & \multirow[t]{2}{*}{$\gamma_{1}$} & \multicolumn{3}{|c|}{ Mode } \\
\hline & & & $\mathrm{I}$ & II & III \\
\hline \multirow[t]{15}{*}{$\mathrm{CCCC}$} & 33 & 0.0 & 35.992 & 73.413 & 73.413 \\
\hline & 34 & 0.0 & 35.986 & 73.395 & 73.395 \\
\hline & 7 & 0.0 & 35.986 & 73.395 & 73.395 \\
\hline & 30 & 0.0 & 35.99 & 73.41 & - \\
\hline & present & & 35.9855 & 73.3954 & 73.3954 \\
\hline & 7 & 0.2 & 39.5097 & 80.5194 & 80.5857 \\
\hline & present & & 39.5097 & 80.5201 & 80.5859 \\
\hline & 7 & 0.4 & 42.9088 & 87.2835 & 87.5259 \\
\hline & 6 & 0.4 & 43.92 & - & - \\
\hline & present & & 42.9087 & 87.2843 & 87.5252 \\
\hline & 35 & 0.5 & 44.696 & - & - \\
\hline & present & & 44.5698 & 90.5565 & 90.9178 \\
\hline & 9 & -0.4 & 28.375 & 57.523 & 57.887 \\
\hline & 6 & -0.4 & 28.424 & - & - \\
\hline & present & & 28.3740 & 57.5240 & 57.8850 \\
\hline \multirow[t]{11}{*}{ SCSC } & 33 & 0.0 & 28.951 & 54.743 & 69.327 \\
\hline & 7 & 0.0 & 28.9509 & 54.7432 & 69.3270 \\
\hline & 36 & 0.0 & 28.9551 & 54.7466 & 69.3393 \\
\hline & 35 & 0.0 & 28.95 & 54.88 & 69.34 \\
\hline & present & & 28.9509 & 54.7431 & 69.3270 \\
\hline & 35 & 0.5 & 36.095 & - & - \\
\hline & present & & 36.9633 & 68.0145 & 85.2326 \\
\hline & 6 & 0.4 & 34.176 & - & - \\
\hline & present & & 34.9521 & 65.4164 & 82.2399 \\
\hline & 6 & -0.4 & 24.052 & - & - \\
\hline & present & & 22.9351 & 43.3774 & 54.0400 \\
\hline \multirow[t]{3}{*}{ FCFC } & 37 & 0.0 & 22.03 & 26.05 & 43.20 \\
\hline & 33 & 0.0 & 22.27 & 26.53 & 43.66 \\
\hline & present & & 22.1922 & 26.4510 & 43.6205 \\
\hline \multirow[t]{8}{*}{ FSFS } & 37 & 0.0 & 9.631 & 16.13 & 36.73 \\
\hline & 33 & 0.0 & 9.6314 & - & - \\
\hline & 11 & 0.0 & 9.631385 & 16.134778 & 36.725643 \\
\hline & present & & 9.6314 & 16.1348 & 36.7256 \\
\hline & 11 & 0.2 & 10.588088 & 17.775345 & 40.383104 \\
\hline & present & & 10.5881 & 17.7753 & 40.3831 \\
\hline & 11 & 0.4 & 11.535708 & 19.460527 & 44.015354 \\
\hline & present & & 11.5357 & 19.4605 & 44.0154 \\
\hline
\end{tabular}

The effect of aspect ratio $a / b$ on the frequency parameter $\Omega$ for $\alpha_{1}=-0.5, \beta_{1}=-0.5, \gamma_{1}=-0.5, \alpha_{2}=-0.5$, $\beta_{2}= \pm 0.5$ and $\gamma_{2}= \pm 0.5$ for the first two modes of vibration has been shown in Fig. 5. It is clear that frequency parameter $\Omega$ increases with the increasing value of $a / b$ for all the boundary conditions, other plate parameters being fixed. The values of $\Omega$ is found to decrease with the increasing value of $\beta_{2}$ and increases with the increasing value of $\gamma_{2}$. The rate of increase in $\Omega$ with $a / b$ is in the order of the boundary conditions FCFC $>$ SCSC $>$ CCCC $>$ FSFS when $\beta_{2}$ changes from -0.5 to 0.5 i.e. the plate is becoming more and more dense towards the edge $Y=1$ and becomes SCSC $>$ CCCC $>$ FCFC $>$ FSFS when $\gamma_{2}$ changes from -0.5 to 0.5 i.e. the plate is becoming more and more stiff towards the edge $Y=1$, keeping all other parameters fixed. This rate of increase is much higher for $a / b>1$ as compared to $a / b<1$ and increases with the increase in the number of modes.

No special feature was observed from the graphs for third mode of vibration (figures not given here) except that the rate of increase/decrease in the frequency parameter $\Omega$ with a specific parameter is higher than that for second mode. Three dimensional mode shapes for specified plate have been plotted using METLAB and shown in Fig. 6.

\section{Conclusions}

The effect of nonhomogeneity arising due to the dependence of Young's modulus and density of the plate material on both the in-plane variables, on the natural frequencies of isotropic rectangular plates of bidirectionally varying 

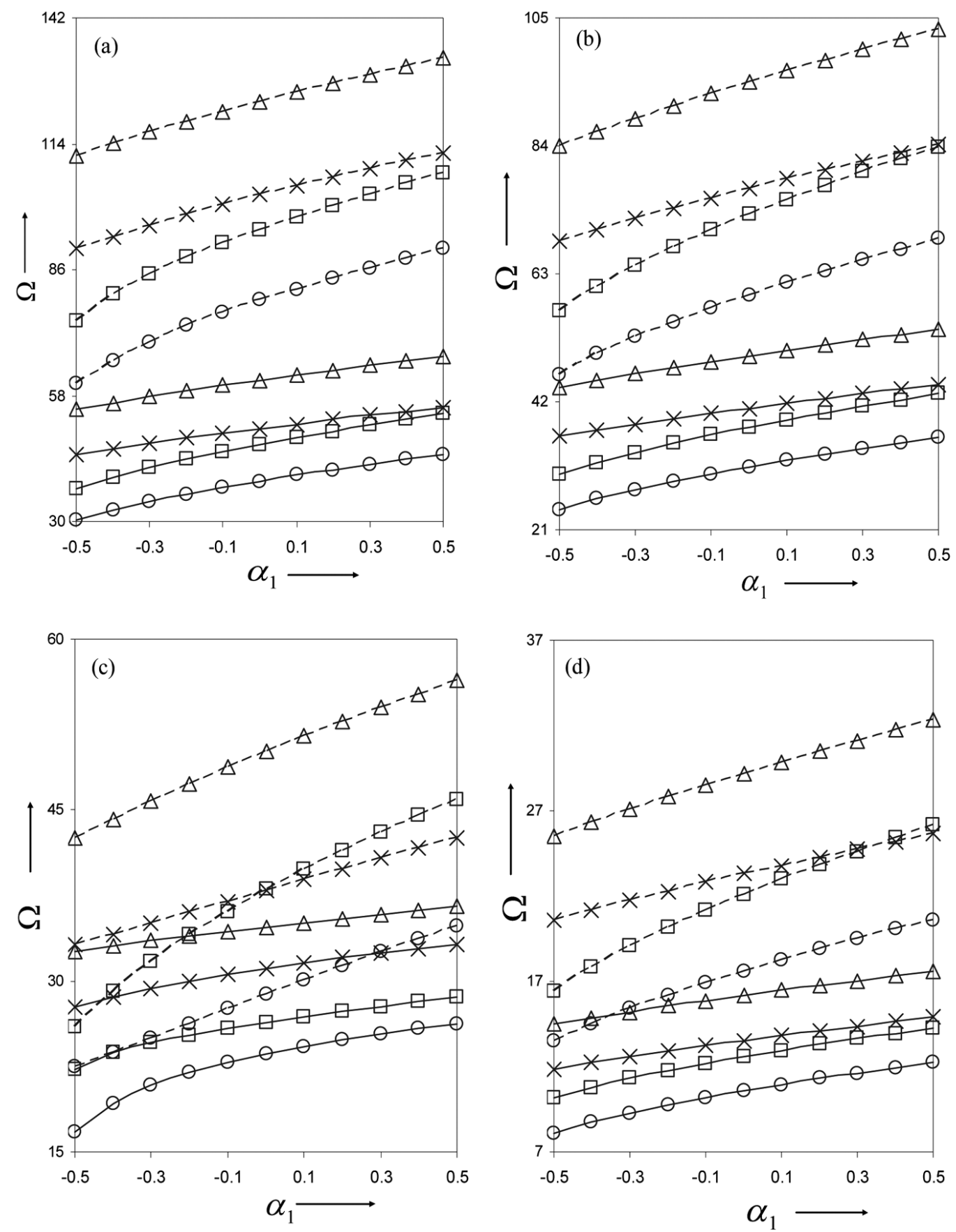

Fig. 2. Frequency parameter $\Omega$ for (a) CCCC, (b) SCSC, (c) FCFC and (d) FSFS plate: for $\beta_{2}=0.5, \gamma_{1}=0.5, \gamma_{2}=0.5$. - , first mode; - - second mode; $\square, \alpha_{2}=-0.5, \beta_{1}=-0.5 ; \mathrm{o}, \alpha_{2}=-0.5, \beta_{1}=0.5 ; \Delta, \alpha_{2}=0.5, \beta_{1}=-0.5 ; \times, \alpha_{2}=0.5, \beta_{1}=0.5$. 

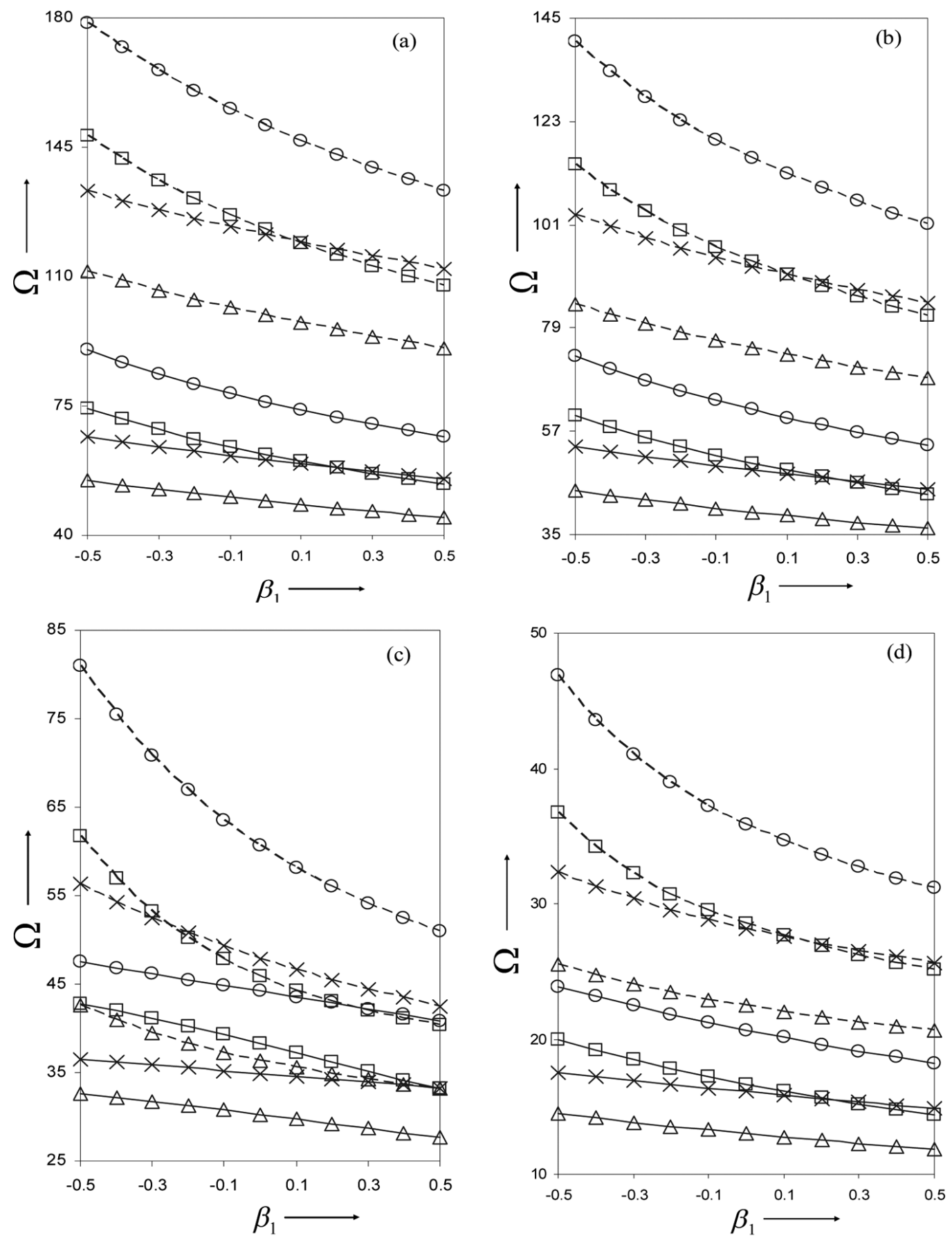

Fig. 3. Frequency parameter $\Omega$ for (a) CCCC, (b) SCSC, (c) FCFC and (d) FSFS plate: for $\alpha_{2}=0.5, \gamma_{1}=0.5, \gamma_{2}=0.5$. - , first mode; - - second mode; $\square, \beta_{2}=-0.5, \alpha_{1}=-0.5 ; \mathrm{o}, \beta_{2}=-0.5, \alpha_{1}=0.5 ; \Delta, \beta_{2}=0.5, \alpha_{1}=-0.5 ; \times, \beta_{2}=0.5, \alpha_{1}=0.5$. 

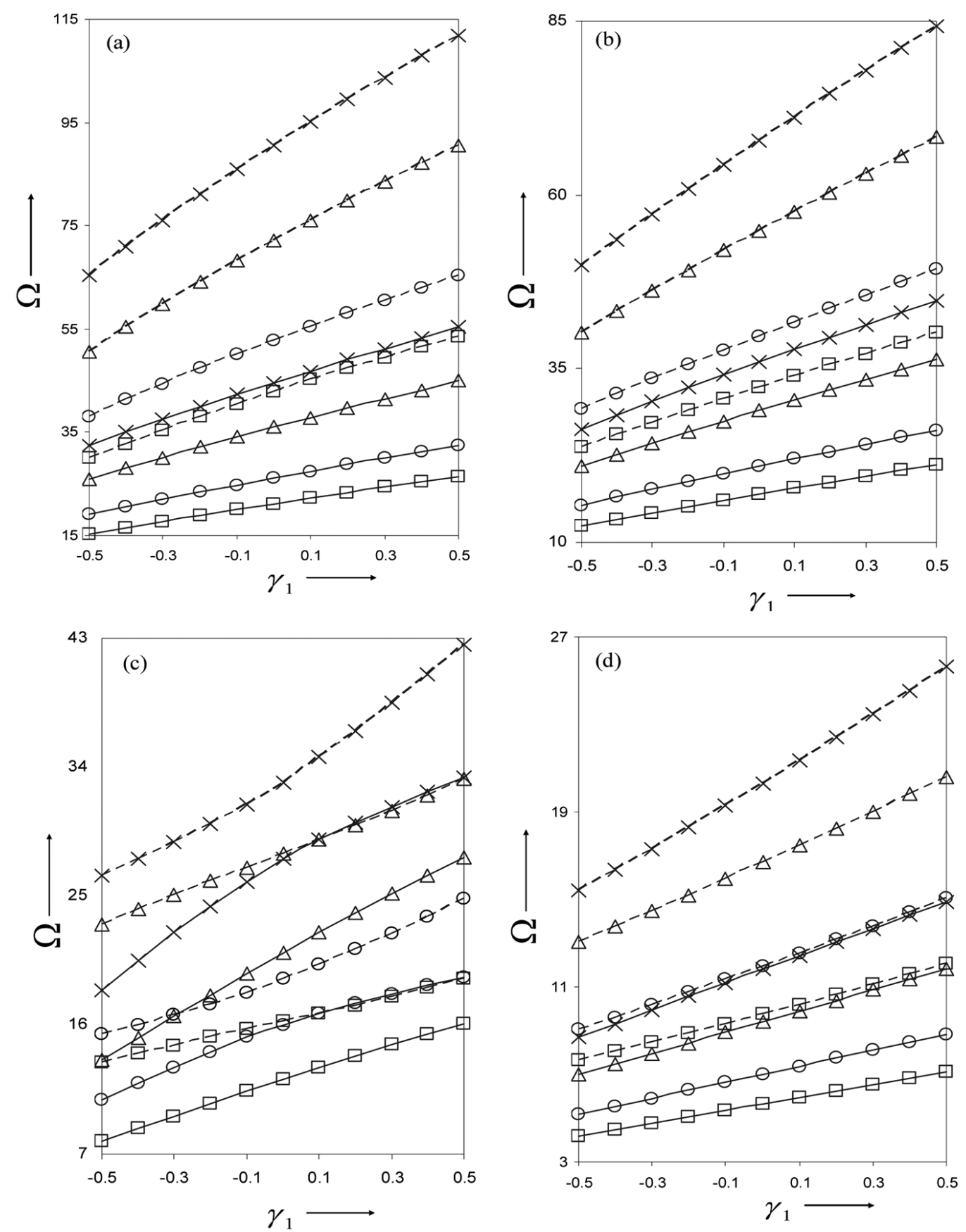

Fig. 4. Frequency parameter $\Omega$ for (a) CCCC, (b) SCSC, (c) FCFC and (d) FSFS plate: for $\alpha_{2}=0.5, \beta_{1}=0.5, \beta_{2}=0.5$. -, first mode; - - - , second mode; $\square, \gamma_{2}=-0.5, \alpha_{1}=-0.5 ; \mathrm{o}, \gamma_{2}=-0.5, \alpha_{1}=0.5 ; \Delta, \gamma_{2}=0.5, \alpha_{1}=-0.5 ; \times, \gamma_{2}=0.5, \alpha_{1}=0.5$. 

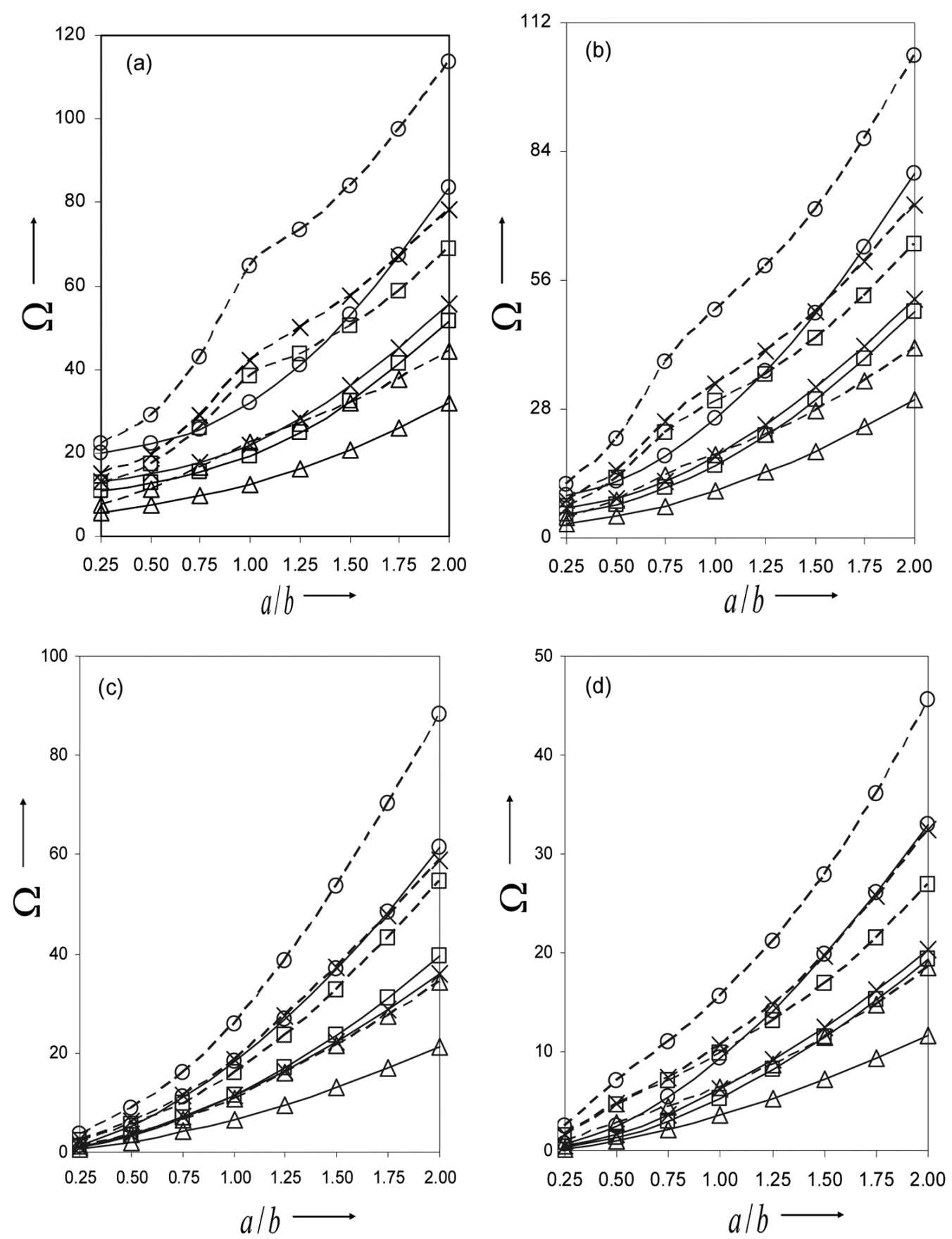

Fig. 5. Frequency parameter $\Omega$ for (a) CCCC,(b) SCSC, (c) FCFC plate,(d) FSFS plate: for $\alpha_{1}=\alpha_{2}=\beta_{1}=\gamma_{1}=-0.5$. - first mode; - -, second mode; $\square, \beta_{2}=-0.5, \gamma_{2}=-0.5 ; \mathrm{o}, \beta_{2}=-0.5, \gamma_{2}=0.5 ; \Delta, \beta_{2}=0.5, \gamma_{2}=-0.5 ; \times, \beta_{2}=0.5, \gamma_{2}=0.5$. 


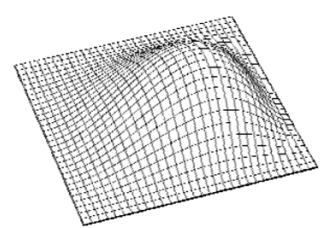

Mode I
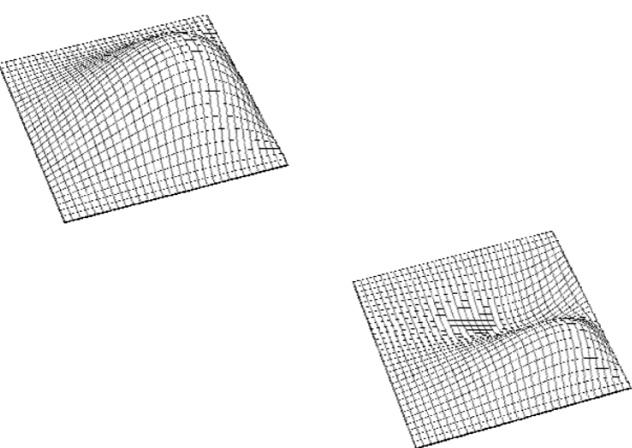

(b)
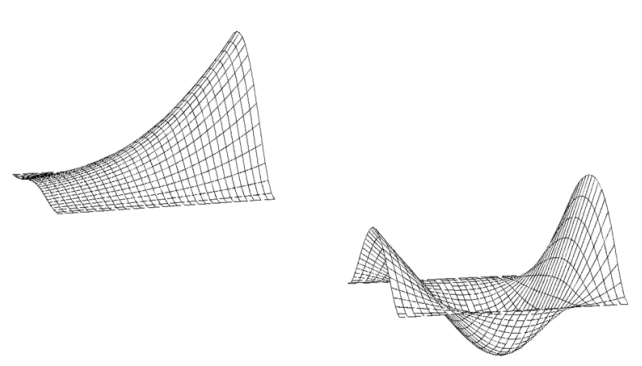

(C)
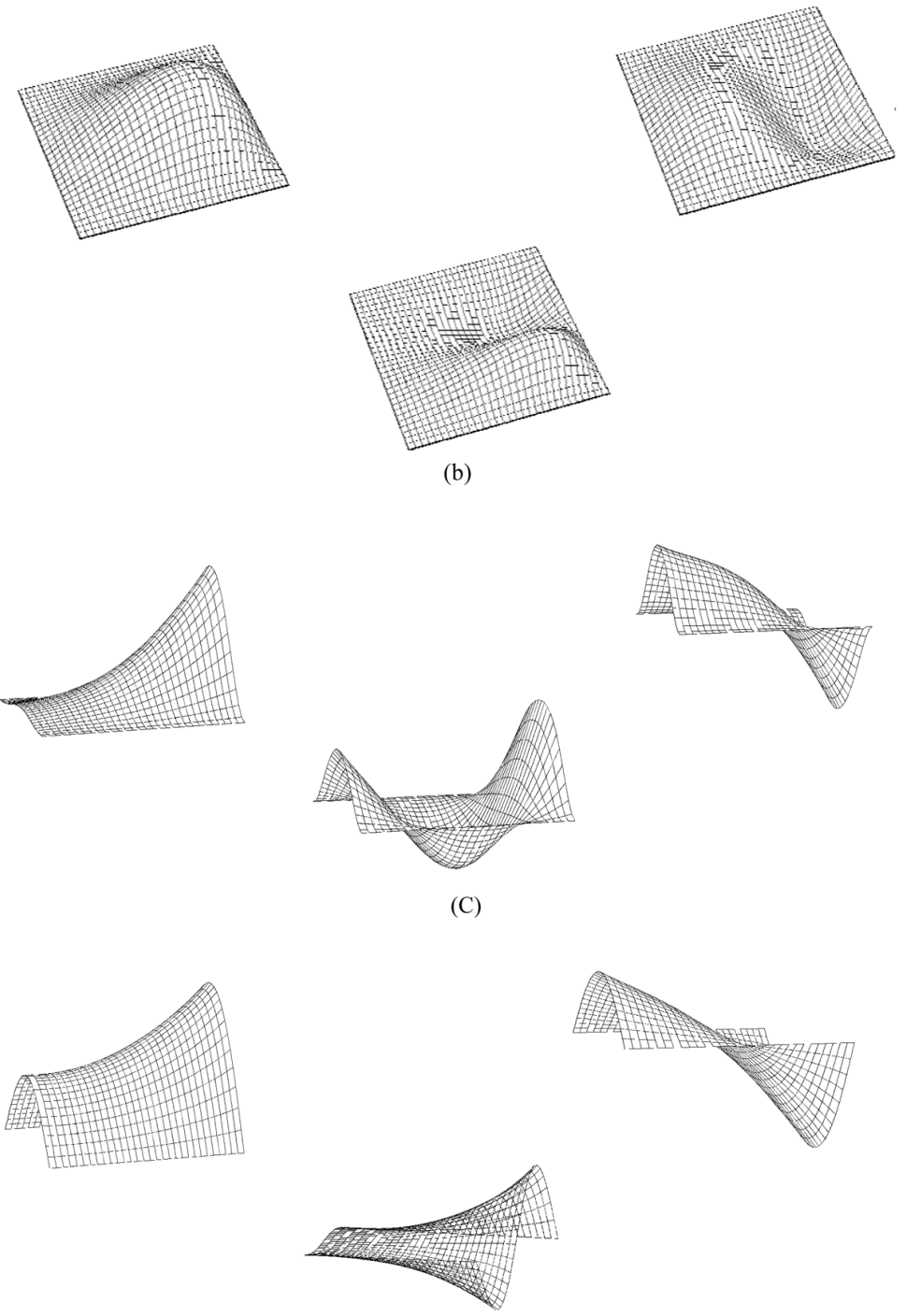

de III

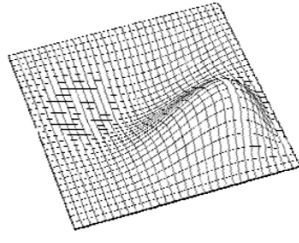

Mode II

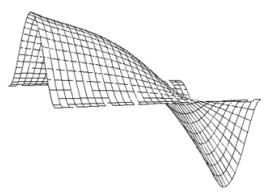

(d)

Fig. 6. First three mode shapes of (a) CCCC, (b) SCSC, (c) FCFC and (d) FSFS square plates for $\alpha_{1}=\beta_{1}=\gamma_{1}=-0.5$ and $\alpha_{2}=\beta_{2}=\gamma_{2}=$ 0.5 . 
thickness has been studied using boundary characteristic orthogonal polynomials in Rayleigh-Ritz method on the basis of classical plate theory. It is observed that the frequency parameter $\Omega$ increases as the plate becomes stiffer and stiffer towards both the edges $X=1$ and $Y=1$ due to an increase in the values of nonhomogeneity parameters $\alpha_{1}$ and $\alpha_{2}$, thickness parameters $\gamma_{1}$ and $\gamma_{2}$, while it decreases as the plate becomes more and more dense towards both the edges $X=1$ and $Y=1$ due to an increase in the values of density parameters $\beta_{1}$ and $\beta_{2}$ for all the four boundary conditions, keeping all other plate parameters fixed. The frequency parameter also increases with the increasing values of aspect ratio $a / b$. The percentage variations in the value of frequency parameter $\Omega$ for the first mode of vibration are -13.9436 to $11.04898,-13.7756$ to $11.1362,-10.9496$ to 7.0636 and -14.6117 to 11.2559 for CCCC, SCSC, FCFC and FSFS boundary conditions, respectively when the nonhomogeneity arises due to the change in only $\alpha_{1}$ from -0.5 to 0.5 . The corresponding variations are -14.1588 to $10.0688,-14.0086$ to 10.0976 , -6.6299 to 7.0057 and -12.3385 to 9.8556 when $\beta_{1}$ changes from -0.5 to 0.5 . These variations decrease by almost $0.5 \%$ with the increase in the number of modes. The present analysis will be of great use to the design engineers dealing with nonhomogeneous plates in obtaining the desired frequency by varying one or more plate parameters considered here.

\section{Acknowledgment}

Authors are thankful to the learned reviewers for their constructive comments. One of the authors, Yajuvindra Kumar is grateful to Council of Scientific and Industrial Research, India for providing the Senior Research Fellowship.

\section{References}

[1] A.W. Leissa, Vibration of Plates (NASA SP 160). Washington D.C.: U.S. Government office, 1969.

[2] A.W. Leissa, Recent research in plate vibrations 1973-1976: Complicating effects, The Shock and Vibration Digest 10(10) (1978), 21-35.

[3] A.W. Leissa, Plate vibration research, 1976-1980: Complicating effects, The Shock and Vibration Digest 13(8) (1981), 19-36.

[4] A.W. Leissa, Recent studies in plate vibrations, 1981-1985 Part I: Classical theory, The Shock and Vibration Digest 19(2) (1987), 11-18.

[5] A.W. Leissa, Recent studies in plate vibrations, 1981-1985 Part II: Complicating effects, The Shock and Vibration Digest 19(3) (1987), 10-24.

[6] H.C. Sanzi, P.A.A. Laura and B.H. Vavergadegreco, Numerical experiments on the determination of the fundamental frequency of transverse vibration of non- uniform rectangular plates, Journal of Sound and Vibration 123(2) (1988), 382-386.

[7] R.B. Bhat, P.A.A. Laura, R.G. Gutierrez, V.H. Cortinez and H.C. Sanzi, Numerical experiments on the determination of natural frequencies of transverse vibrations of rectangular plates of non-uniform thickness, Journal of Sound and Vibration 138(2) (1990), $205-219$.

[8] P.M. Bells, M.J. Maurizi, P.A.A. Laura and H.C. Sanzi, Free vibration of rectangular plates of exponentially varying thickness and with two free edges, Ocean Engineering 19(4) (1992), 405-408.

[9] K.M. Liew and M.K. Lim, Transverse vibration of trapezoidal plates of variable thickness: symmetric trapezoids, Journal of Sound and Vibration 165(1) (1993), 45-67.

[10] R.K. Roy and N. Ganesan, Studies on the dynamic behavior of a square plate with varying thickness, Journal of Sound and Vibration 182(3) (1995), 205-219.

[11] C.W. Bert and M. Malik, Free vibration analysis of tapered rectangular plates by differential quadrature method: a semi-analytical approach, Journal of Sound and Vibration 190(1) (1996), 41-63.

[12] A.Ya. Grigorenko and T.V. Tregubenko, Numerical and experimental analysis of rectangular plates with variable thickness, International Applied Mechanics 36 (2000), 268-270.

[13] K.M. Liew, T.Y. Nag and S. Kitipornchai, A semi-analytical solution for vibration of rectangular plates with abrupt thickness variation, International Journal of Solids and Structures 38(28-29) (2001), 4937-4954.

[14] R. Szilard, Theories and Applications of Plate Analysis: classical, numerical and engineering methods, John Wiley and Sons, 2004.

[15] S.W. Kang and S.-H. Kim, Vibration analysis of simply supported rectangular plates with unidirectionally, arbitrarily varying thickness, Journal of Sound and Vibration 312(4-5) (2008), 551-562.

[16] P.A.A. Laura and R.O. Grossi, Transverse vibrations of rectangular plates with thickness varying in two directions and with edges elastically restrained against rotation, Journal of Sound and Vibration 63(4) (1979), 499-505.

[17] R.O. Grossi and P.A.A. Laura, Transverse vibrations of orthotropic rectangular plates with thickness varying in two directions and with edges elastically restrained against rotation, Fibre Science and Technology 14(4) (1980-1981), 311-317.

[18] B. Singh and V. Saxena, Transverse vibration of a rectangular plate with bidirectional thickness variation, Journal of Sound and Vibration 198(1) (1996), 51-65.

[19] T. Sakiyama and M. Huang, Free vibration analysis of rectangular plates with variable Thickness, Journal of Sound and Vibration 216(3) (1998), 379-397.

[20] Y.K. Cheung and D. Zhou, The free vibrations of tapered rectangular plates using a new set of beam functions with the Rayleigh-Ritz method, Journal of Sound and Vibration 223(5) (1999), 703-722. 
[21] P. Malekzadeh and S.A. Shahpari, Free vibration analysis of variable thickness thin and moderately thick plates with elastically restrained edges by DQM, Thin Walled Structures 43(7) (2005), 1037-1050.

[22] M. Huang, X.Q. Ma, T. Sakiyama, H. Matsuda and C. Morita, Free vibration analysis of orthotropic rectangular plates with variable thickness and general boundary conditions, Journal of Sound and Vibration 288(4-5) (2005), 931-955.

[23] M. Huang, X.Q. Ma, T. Sakiyama, H. Matsuda and C. Morita, Free vibration analysis of rectangular plates with variable thickness and point support, Journal of Sound and Vibration 300(3-5) (2007), 435-452.

[24] R. Lal and Dhanpati, Transverse vibrations of non-homogeneous orthotropic rectangular plates of variable thickness: A spline technique, Journal of Sound and Vibration 306(1-2) (2007), 203-214.

[25] R. Lal and Dhanpati, Quintic splines in the study of buckling and vibration of non-homogeneous orthotropic rectangular plates with variable thickness, International Journal of Applied Mathematics and Mechanics 3(3) (2007), 18-35.

[26] R. Lal, Dhanpati and Y. Kumar, Buckling and vibrations of non-homogeneous orthotropic rectangular plates of varying thickness under biaxial compression, International Journal of Applied Mathematics and Mechanics 4(4) (2008), 93-107.

[27] R. Lal and Dhanpati, Effect of nonhomogeneity on vibration of orthotropic rectangular plates of varying thickness resting on Pasternak foundation, Journal of Vibration and Acoustics 131 (2009), 011001-7.

[28] B. Singh and S. Chakraverty, Flexural vibration of skew plates using boundary characteristic orthogonal polynomials in two variables, Journal of Sound and Vibration 173(2) (1994), 157-178.

[29] T.S. Chihara, An Introduction to Orthogonal Polynomials, Gordan and Breach, London, 1978.

[30] K.M. Liew, K.Y. Lam and S.T. Chow, Free vibration analysis of rectangular plates using orthogonal plate function, Computers and Structures 34(1) (1990), 79-85.

[31] R.B. Bhat, Flexural vibration of polygonal plates using characteristic orthogonal polynomials in two variables, Journal of Sound and Vibration 114 (1987), 65-71.

[32] G.H. Golub and C.F. Van Loan, Matrix Computations, (3rd Edition), Baltimore, John Hopkins, 1996.

[33] A.W. Leissa, Free vibrations of rectangular plates, Journal of Sound and Vibration 31(3) (1973), 257-293.

[34] R.B. Bhat, Natural frequencies of rectangular plates using characteristic orthogonal polynomials in Rayleigh-Ritz method, Journal of Sound and Vibration 102(4) (1985), 493-499.

[35] P.A.A. Laura, H.A. Larrondo, V.H. Cortinez and D.R. Avalos, Transverse vibrations of rectangular plates of non-uniform thickness subjected to a uniform state of in-plane stress, Journal of Sound and Vibration 151(1) (1991), 175-180.

[36] W. Yan and W. Zhong-min, Transverse vibration of viscoelastic rectangular plate with linearly varying thickness and multiple cracks, Journal of Sound and Vibration 318(4-5) (2008), 1005-1023.

[37] T. Mizusawa, Natural frequencies of rectangular plates with free edges, Journalof Sound and Vibration 105(3) (1986), 451-459. 

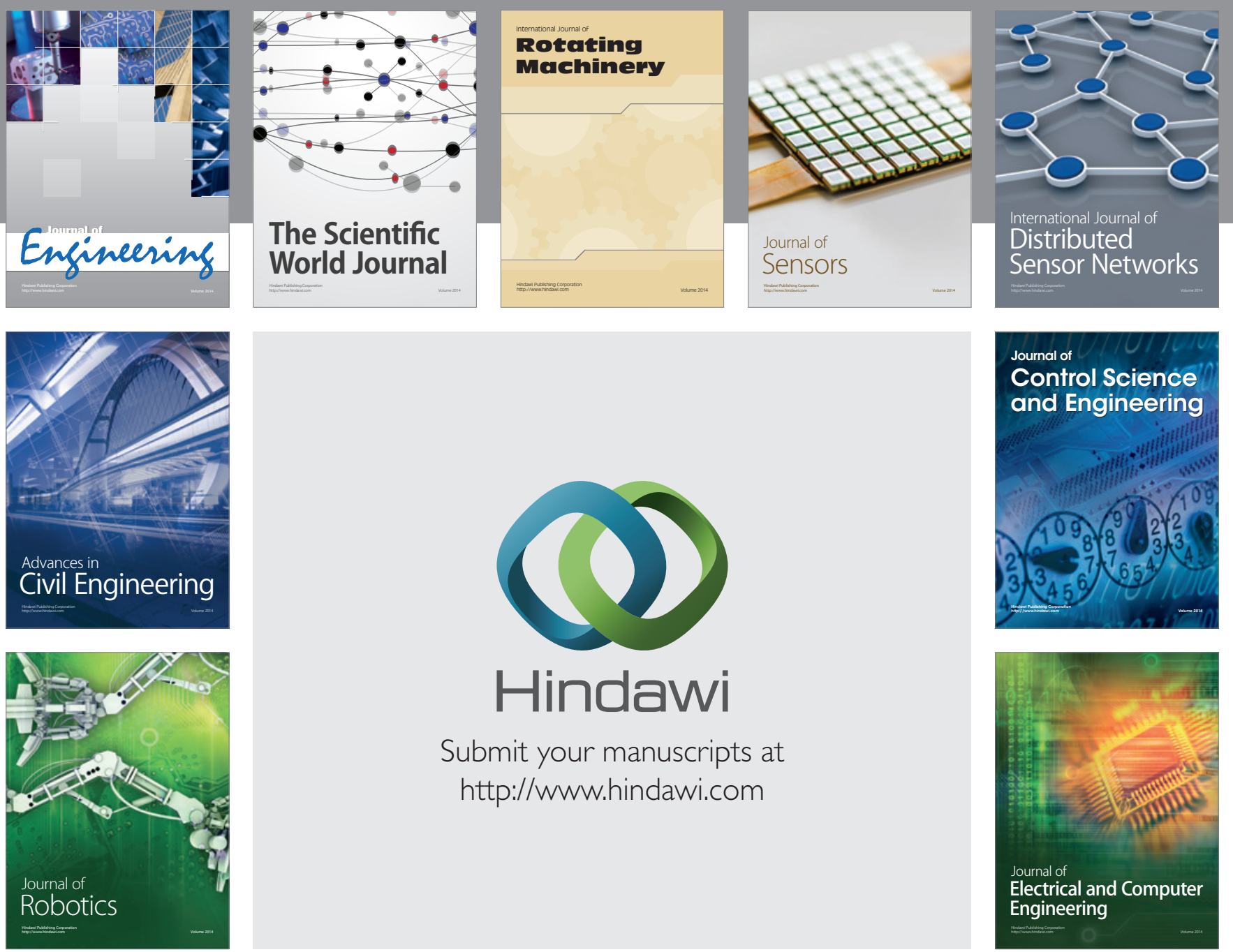

Submit your manuscripts at

http://www.hindawi.com
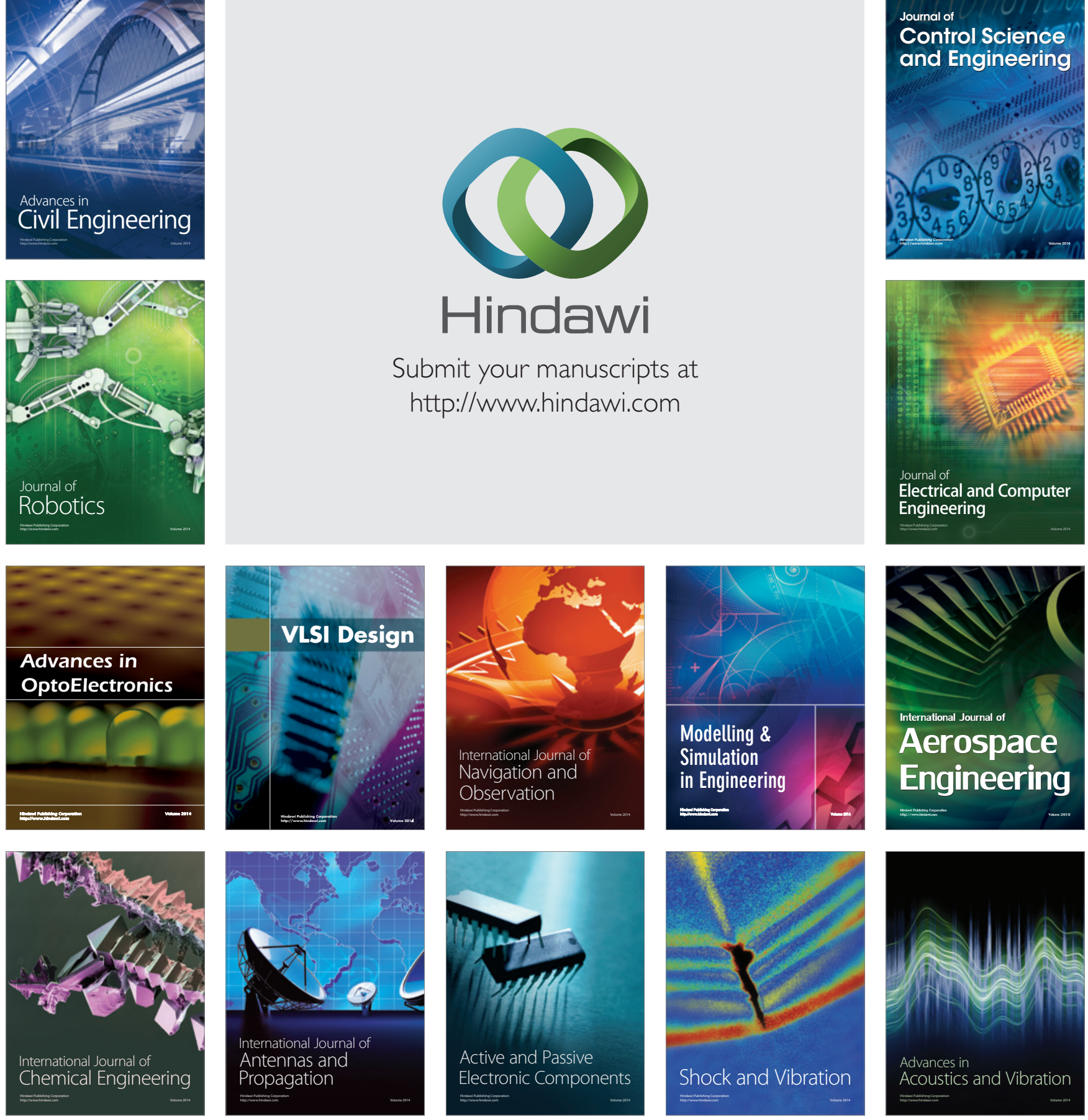